\title{
Anti-gout Preparation
}

National Cancer Institute

\section{Source}

National Cancer Institute. Anti-gout Preparation. NCI Thesaurus. Code C29733.

Pharmaceutical Anti-gout Preparations such as colchicine, allopurinol, probenecid, and sulfinpyrazone contain natural or synthetic agents against gout, a condition characterized by increased uric acid accumulation. Anti-gout agents may reduce inflammation, stimulate elimination of uric acid, or increase production of uric acid.

$(\mathrm{NCl04})$ 